\section{Gastrostomy Tube Placement Outcomes in Children: Comparison of Open and Laparoscopic Methods}

\section{Abstract}

Background: Although gastrostomy tube placement in children is increasingly performed and laparoscopic gastrostomy tube insertions considered by many surgeons to be the "gold standard", however, there is no definitive data that proves the benefits of laparoscopic technique over the open. This study aimed to compare two methods and clinical outcomes between patients undergoing laparoscopic and open gastrostomy tube insertion

A retrospective study data was entered and analyzed through statistical package SPSS version 22 conducted to identify inpatient hospitalizations for gastrostomy placements for the treatment of gastro esophageal reflux disease, feeding intolerance and swallowing disorder (2007-2016) at King Fahad Medical City in Riyadh, Saudi Arabia. The outcomes evaluated using ANOVA test comparing the indications for the procedure, concomitant diseases and postoperative complications associated with both techniques. Because ANOVA is a data set that evaluates the mean significant difference between operative methods and other study parameters, a single-institution retrospective study was conducted in which each technique compared to the other during the same period. Outcome measures (institutional data) were used to compare rates of postoperative complications in terms of leakage, ileus, nausea, diarrheae, pain after feeding, high residual, granulation tissue formation and tube revision between the two cohorts.

Results: Has been evaluated 180 patients with gastrostomy tube placement (94 open vs. 86 laparoscopic), 44 with concomitant fundoplication and with 3 different types of gastrostomy tubes used during the procedures. There were differences in gender (male to female 1:2), but not in age distribution, or comorbidity between the two groups. Compared with open technique of gastrostomy placement, the postoperative complications in terms of postoperative nausea $20(21.5 \%)$, pain after feeding $6(6.4 \%)$ and leakage $25(26.6 \%)$ were more with open technique versus $4(4.7 \%) p=0.006,0(0.0 \%) p=0.017$ and $16(18.6 \%) p=0.202$ respectively in laparoscopic technique. Concomitant fundoplication as more commonly performed for patients with neurological disorders in laparoscopic technique 29 (33.7\%) vs. open $15(16.0 \%) p=0.006$. Postoperative diarrheae was observed more in patients who underwent Mic-Key tube placement $p 0.008$, however length of the hospital stay was more after Mic tube placement $p=001$. The institutional data included outcome of patients with neurological disorders who underwent gastrostomy tube placement $21(60.0 \%)$ with sequel and $113(80.3 \%)$ without sequel and neurological disorders $\mathrm{p}=0.015$.

Conclusion: Although the technical and clinical outcomes for open and laparoscopic tube placement appear comparable, laparoscopic technique is associated with shorter length of stay and fewer complications.

Keywords: Gastrostomy tubes, Reflux, leakage, Laparoscopy

\section{Abdulwahhab AlJubab, Illhama A Jafarli, Tariq AlTokhais, Lubna Abdallah, Osama Mosallam, Reem AlJubab, Mohammed Bashir Salma
and Nouri Ourfali}

Department of Pediatric Surgery, King Fahad Medical City, Saudi Arabia

Corresponding author:

Abdulwahhab AlJubab

झ aaljubab@kfmc.med.sa

Head of Pediatric Surgery department, King Fahad Medical City, pediatric surgery, Saudi Arabia.

Tel: 00966-11-2889999

Citation: AlJubab A, Jafarli IA, AlTokhais $\mathrm{T}$, et al. Gastrostomy Tube Placement Outcomes in Children: Comparison of Open and Laparoscopic Methods. J Univer Surg. 2017, 5:3. 


\section{Introduction}

Gastrostomy tube placement in children remains one of the interventions performed by surgeons, gastrointestinal endoscopists and interventional radiologists. 3 single-center retrospective reviews have been conducted which revealed different outcomes following open, percutaneous endoscopic and laparoscopic gastrostomy tube insertion in children, reporting varying results [1]. Gastrostomy feeding tube placement in children is associated with a high frequency of adverse events. This study sought to preoperatively estimate postoperative adverse events in children undergoing gastrostomy feeding tube placement [2].

Four different technical approaches can be employed for gastrostomy tubes insertion, using one of: surgically the Stamm or open technique (OPEN), the percutaneous-endoscopic approach (PEG), guided by interventional-radiology (IRG) or by laparoscopic (LAP) minimally invasive surgery. The original description of the surgical technique of placing a gastrostomy tube was provided in 1984 by Stamm. The placement of a gastrostomy tube in a pediatric patient often represents a crucial moment for the child, his/her family and the medical team providing care. The implications of the procedure resonate for years to follow as care plans shift to outpatient management and nutritional needs [3]. One of the major advantages of the addition of laparoscopy in comparison to a percutaneous endoscopic approach is the improved visualization of the abdominal cavity, which aids in minimizing the risk of injury of the surrounding structures [4]. Also surgical gastrostomy insertion in children $<1$ year of age yielded the greatest increase in number over the last years [5].

Numerous studies have shown that laparoscopic gastrostomy tube placement is a relatively simple and safe alternative to standard open surgical technique or percutaneous endoscopic management because it obviates the need for prolonged hospital stay and lessens the complications rate. In a pooled analysis of gastrostomy tube insertions, the rates of technical success and clinical effectiveness in laparoscopic versus percutaneous techniques as well as complication rates after percutaneous endoscopic gastrostomies were reported to be greater than $77.4 \%$ [6]. Because all clinical studies were from single centers and included a small cohort of patients, the general applicability of these data is debatable.

The objectives of the current study were to compare the clinical outcomes and postoperative complications at a patient level by conducting a single-institution retrospective study for patients undergoing laparoscopic versus open surgical gastrostomy tube insertion.

\section{Materials and Methods}

This study was executed in two parts. First, a retrospective study was conducted to evaluate patient outcomes at an institutional level. Second, data was used to evaluate length of stay for patients who underwent laparoscopic or open gastrostomy tube insertion.

\section{Data source}

A retrospective analysis was conducted using the 2007-2016 data in King Fahad Medical City, Riyadh, Saudi Arabia. The database contains complete inpatient hospitalization records for the entire hospital. These claims report patient demographic information such as age, sex, diagnosis, and comorbidities. In addition, information regarding the hospitalization is provided including length of stay, diagnostic testing, and therapeutic procedures.

\section{Patient population}

The study population consisted of all unique hospitalization claims for the placement of a laparoscopic or open gastrostomy tube insertion procedure. Claims were included based on the primary diagnosis and procedure. A retrospective analysis was conducted with consecutive patients ( $>1$ years of age) who underwent surgical, open versus laparoscopic technique for management of gastro esophageal reflux disease, feeding intolerance and swallowing disorder. The inclusion criteria specified patients older than 1 year old with an underlying diagnosis of gastro esophageal reflux disease, feeding intolerance or swallowing disorder who had undergone procedures for relief of the relative condition. The exclusion criteria ruled out patients who responded to conservative treatment with ant reflux medications with normal swallowing pattern, patients with postoperative granulation tissue at the tube insertion site as the rate it was identical in both techniques and children below 1 year old.

For each patient treated with open gastrostomy tube insertion one patient who underwent laparoscopic approach of the same procedure were matched by an independent observer for the following variables: leakage, ileus, nausea, diarrhea, pain after feeding, high residual, and tube revision. The medical records of all the study subjects were reviewed for patient demographics, clinical presentation, comorbidities, laboratory investigations, and radiologic investigations

\section{Outcome Measures}

The rates for treatment success, complications, and reinterventions were compared as well as the length of the post procedure hospital stay between each treatment method

\section{Open Stamm gastrostomy tube insertion}

Incision performed through a small $(6-8 \mathrm{~cm})$ upper midline incision. The abdominal cavity is entered with care taken to take down any adhesions to the inner abdominal wall with gentle traction. Babcock clamps are used to grasp the anterior stomach wall in the mid to slightly distal stomach and to elevate it into the wound. A circular purse-string suture is placed with a diameter of $1.5-2.0 \mathrm{~cm}$ using a 2-0 or 3-0 suture with the ends left untied. A concentric purse-string of 2-0 or 3-0 silk is then placed just outside of the first purse-string with the ends left untied. A small opening is made in the serosa of the stomach in the center of the two concentric purse-string sutures. A 14-18 French Foley balloon catheter is then placed into the stomach through the just made opening. 


\section{Laparoscopic gastrostomy tube insertion}

Contraindications to laparoscopic gastrostomy tube placement included those who cannot tolerate pneumoperitoneum (severe pulmonary or cardiac disease), active skin infection, acute illness, or suboptimal treatment of chronic illness as this is not an emergent or urgent procedure. An incision is made in the umbilicus and a 3-5 mm port is placed. Another $3 \mathrm{~mm}$ stab incision may be made in the right upper quadrant or for further dissection/retraction. The stomach is insufflated with air. An appropriate place on the stomach is selected as the gastrostomy tube site was two thirds from the gastro esophageal junction to the pylorus. Care was taken to ensure that the gastrostomy tube is not too close to the pylorus as the balloon on the tube can case pyloric obstruction. The stomach is grasped with the locking grasper in the location where the gastrostomy tube is to be placed. Two securing sutures are then placed which will secure the stomach to the anterior abdominal wall. The gastrostomy tube was then placed between these two sutures. After the gastrostomy tube was in place, secured in place with a dressing.

\section{Statistical analysis}

All analyses were performed using statistical package Chisquare/Fisher's exact test according to whether the cell expected frequency is smaller than 5 and it was used to determine the significant relationship among categorical variables.

\section{Institutional data}

A single-institution retrospective case-control study was conducted in which each patient who underwent a laparoscopic placement of gastrostomy tube was matched with one who underwent open technique during the same period (20072016). Statistical analysis was performed using SPSS version 22. All Categorical variables gender, nationality, previous history etc. were presented as numbers and percentages. Continuous variables age, weight, operative time, albumin level etc. were expressed as Mean \pm Standard Deviation. Independent sample t-test/ANOVA was applied to evaluate the mean significant difference between outcome and other study parameters. A chisquare test was used to compare the proportions of basic clinical characteristics of the patients (Table 1) and outcome measures (Table 2 ) across the two groups.

\section{Results}

A total of 180 patients with gastrostomy tube placement (94 open vs. 86 laparoscopic), 44 with concomitant fundoplication and with 3 different types of gastrostomy tubes used during the procedures. The age, gender, and comorbidities of the reported patients in association with the type of the tubes and outcome of the study in both cohorts are shown in (Tables 3 and 4).

\section{Health resource use}

The median aggregate hospital days or the total length of stay from admission to discharge was $6.99 \pm 0.96$ days for Foley catheter placement, $9.49 \pm 1.99$ days for Mickey tube and $46.09 \pm 3.75$ days for Mic tube placement $(p<0.001)$. Operative
Table 1 Basic Clinical characteristics of Patients $(n=180)$.

\begin{tabular}{|c|c|c|}
\hline Variables & Categories & $n(n \%)$ \\
\hline \multirow{2}{*}{ Gender } & Male & $111(61.7 \%)$ \\
\hline & Female & $69(38.3 \%)$ \\
\hline \multirow{2}{*}{ Nationality } & Saudi & $177(98.3 \%)$ \\
\hline & Non - Saudi & $3(1.7 \%)$ \\
\hline \multirow{2}{*}{ Previous Abnormal Surgery } & Yes & $6(3.3 \%)$ \\
\hline & No & $174(96.7 \%)$ \\
\hline \multirow{2}{*}{ Concomitant Fundoplication } & Yes & $44(24.4 \%)$ \\
\hline & No & $136(75.6 \%)$ \\
\hline \multirow{4}{*}{ Indication of the procedure } & GERD & $51(28.3 \%)$ \\
\hline & Feeding Intolerance & $6(3.3 \%)$ \\
\hline & Swallowing disorder & $40(22.2 \%)$ \\
\hline & More than one indications & $83(46.1 \%)$ \\
\hline \multirow{2}{*}{ Procedure type } & Open & $94(52.2 \%)$ \\
\hline & Laparoscopic & $86(47.8 \%)$ \\
\hline \multirow{3}{*}{ Neurological disorder } & Yes & $139(77.2 \%)$ \\
\hline & No & $41(22.8 \%)$ \\
\hline & ASAI & $11(6.1 \%)$ \\
\hline \multirow{4}{*}{ ASA Status } & ASA II & $62(34.4 \%)$ \\
\hline & ASAIII & $106(58.9 \%)$ \\
\hline & ASAE & $1(0.6 \%)$ \\
\hline & Foley Cath & $113(62.8 \%)$ \\
\hline \multirow[t]{2}{*}{ Type of GT used } & Mickey Tube & $56(31.1 \%)$ \\
\hline & Mic Tube & $11(6.1 \%)$ \\
\hline \multirow{2}{*}{ Post-Operative Complications } & Yes & $59(32.8 \%)$ \\
\hline & No & $121(67.2 \%)$ \\
\hline \multirow{2}{*}{ ileus } & Yes & 7 (3.9\%) \\
\hline & No & $173(96.1 \%)$ \\
\hline \multirow{2}{*}{ diarrhea } & Yes & $27(15.0 \%)$ \\
\hline & No & $153(85.0 \%)$ \\
\hline \multirow{2}{*}{ nausea } & Yes & $24(13.3 \%)$ \\
\hline & No & $156(86.7 \%)$ \\
\hline \multirow{2}{*}{ pain after feeding } & Yes & $6(3.3 \%)$ \\
\hline & No & $174(96.7 \%)$ \\
\hline \multirow{2}{*}{ leakage } & Yes & $41(22.8 \%)$ \\
\hline & No & $139(77.2 \%)$ \\
\hline \multirow{2}{*}{ granuloma } & Yes & 151 (83.8\%) \\
\hline & No & $29(16.1 \%)$ \\
\hline \multirow{2}{*}{ tube revision } & Yes & $10(5.6 \%)$ \\
\hline & No & 170 (94.4\%) \\
\hline \multirow{2}{*}{ high residual } & Yes & $13(7.2 \%)$ \\
\hline & No & $167(92.8 \%)$ \\
\hline \multirow{3}{*}{ Outcome } & Death & $5(2.8 \%)$ \\
\hline & Full Recovery & $140(77.8 \%)$ \\
\hline & Recovery with sequelae & 35 (19.4\%) \\
\hline
\end{tabular}

time for open technique was less $84.11 \pm 9.45$ minutes than for laparoscopic $90.18 \pm 10.30$ minutes $(p=0.665)$ due to the concomitant fundoplication applied with laparoscopic approach more 29 (33.7\%) than with open technique 15 (16.0\%) ( $p=0.006)$. However, feeding for the patients who underwent laparoscopic technique started from the first postoperative day with delayed feeding on day 2 or 3 for patients on who performed the open technique. 
Table 2 Association between type of procedure and clinical characteristics of the patient.

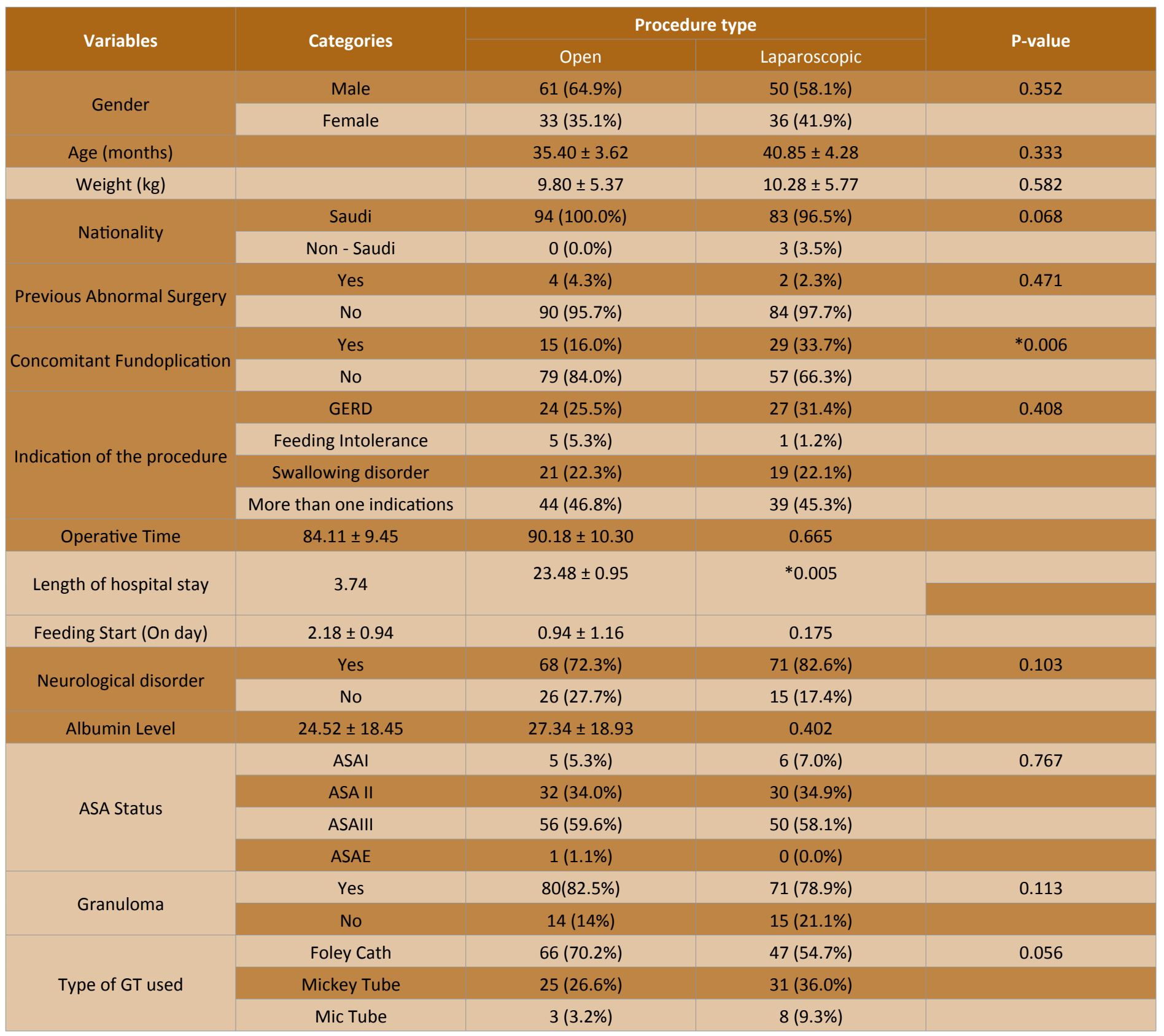

\section{Institutional data}

(Tables 1 and 2) displays the demographics and disease characteristics of all the patients at presentation. The two cohorts did not differ significantly in terms of patient demographics, type of procedure and clinical characteristics of the patient or comorbidities.

Compared with open technique of gastrostomy placement, the postoperative complications in terms of postoperative nausea 20 (21.5\%), pain after feeding $6(6.4 \%)$ and leakage $25(26.6 \%)$ were more with open technique versus $4(4.7 \%) p=0.006,0(0.0 \%)$ $\mathrm{p}=0.017$ and $16(18.6 \%)$ p 0.202 respectively in laparoscopic technique. Concomitant fundoplicaiton as more commonly performed for patients with neurological disorders in laparoscopic technique 29 (33.7\%) vs. open $15(16.0 \%) p=0.006$. Postoperative diarrheae was observed more in patients who underwent MicKey tube placement $p=0.008$, however length of the hospital stay was more after Mic tube placement $p=001$. The institutional data included outcome of patients with neurological disorders who underwent gastrostomy tube placement 21 (60.0\%) with sequel and $113(80.3 \%)$ without sequel and neurological disorders $\mathrm{p}=0.015$.

\section{Discussion}

Placement of a gastrostomy tube for feeding is one of the most commonly performed pediatric surgery procedures. The options for feeding tube placement into the stomach include an open gastrostomy tube placement, a laparoscopic gastrostomy tube placement, a percutaneous endoscopic gastrostomy (PEG), and a laparoscopic-assisted percutaneous endoscopic gastrostomy 
Table 3 Association between type of tube and clinical characteristics of the patient.

\begin{tabular}{|c|c|c|c|c|c|}
\hline & & \multicolumn{3}{|c|}{ Outcome of the study } & \multirow[b]{2}{*}{$P$ - Value } \\
\hline & & Death & Full Recovery & Recovery & \\
\hline Variables & Parameters & & with sequelae & & \\
\hline \multirow[t]{2}{*}{ Gender } & Male & $0(0.0 \%)$ & $88(62.9 \%)$ & $23(65.7 \%)$ & $* 0.015$ \\
\hline & Female & $5(100.0 \%)$ & $52(37.1 \%)$ & $12(34.3 \%)$ & \\
\hline Age (months) & $48.00 \pm 30.1$ & $40.27 \pm 3.18$ & $27.52 \pm 5.01$ & 0.164 & \\
\hline Weight (kg) & $5.3 \pm 2.46$ & $10.28 \pm 0.47$ & $9.76 \pm 1.05$ & 0.138 & \\
\hline \multirow[t]{2}{*}{ Nationality } & Saudi & $5(100.0 \%)$ & $137(97.9 \%)$ & $35(100.0 \%)$ & 0.647 \\
\hline & Non - Saudi & $0(0.0 \%)$ & $3(2.1 \%)$ & $0(0.0 \%)$ & \\
\hline \multirow[t]{2}{*}{ Previous Abnormal Surgery } & Yes & $0(0.0 \%)$ & $5(3.6 \%)$ & $1(2.9 \%)$ & 0.895 \\
\hline & No & $5(100.0 \%)$ & 135 (96.4\%) & $34(97.1 \%)$ & \\
\hline \multirow[t]{2}{*}{ Concomitant Fundoplication } & Yes & $0(0.0 \%)$ & $39(27.9 \%)$ & $5(14.3 \%)$ & 0.108 \\
\hline & No & $5(100.0 \%)$ & $101(72.1 \%)$ & $30(85.7 \%)$ & \\
\hline \multirow[t]{4}{*}{ Indication of the procedure } & GERD & $0(0.0 \%)$ & $38(27.1 \%)$ & $13(37.1 \%)$ & 0.277 \\
\hline & Feeding Intolerance & $0(0.0 \%)$ & $6(4.3 \%)$ & $0(0.0 \%)$ & \\
\hline & Swallowing disorder & $2(40.0 \%)$ & $34(24.3 \%)$ & $4(11.4 \%)$ & \\
\hline & More than one indications & $3(60.0 \%)$ & $62(44.3 \%)$ & $18(51.4 \%)$ & \\
\hline Operative Time & $60.00 \pm 0.1$ & $89.44 \pm 7.96$ & $76.75 \pm 13.91$ & 0.683 & \\
\hline Feeding Start (On day) & $2.00 \pm 0.3$ & $2.03 \pm 1.06$ & $2.29 \pm 1.14$ & 0.595 & \\
\hline \multirow[t]{2}{*}{ Neurological disorder } & Yes & $5(100.0 \%)$ & $113(80.7 \%)$ & $21(60.0 \%)$ & $* 0.015$ \\
\hline & No & $0(0.0 \%)$ & $27(19.3 \%)$ & $14(40.0 \%)$ & \\
\hline Albumin Level & $26.6 \pm 9.4$ & $25.29 \pm 1.87$ & $28.4 \pm 4.01$ & 0.789 & \\
\hline \multirow[t]{4}{*}{ ASA Status } & ASAI & $0(0.0 \%)$ & $9(6.4 \%)$ & $2(5.7 \%)$ & 0.39 \\
\hline & ASA II & $2(40.0 \%)$ & $42(30.0 \%)$ & $18(51.4 \%)$ & \\
\hline & ASAIII & $3(60.0 \%)$ & $88(62.9 \%)$ & $15(42.9 \%)$ & \\
\hline & ASAE & $0(0.0 \%)$ & $1(0.7 \%)$ & $0(0.0 \%)$ & \\
\hline \multirow[t]{2}{*}{ Post-Operative Complications } & Yes & $5(100.0 \%)$ & $40(28.6 \%)$ & $14(40.0 \%)$ & $* 0.002$ \\
\hline & No & $0(0.0 \%)$ & $100(71.4 \%)$ & $21(60.0 \%)$ & \\
\hline \multirow[t]{2}{*}{ ileus } & Yes & $0(0.0 \%)$ & $5(3.6 \%)$ & $2(5.7 \%)$ & 0.759 \\
\hline & No & $5(100.0 \%)$ & $135(96.4 \%)$ & $33(94.3 \%)$ & \\
\hline \multirow[t]{2}{*}{ diarrhea } & Yes & $0(0.0 \%)$ & $23(16.4 \%)$ & $4(11.4 \%)$ & 0.483 \\
\hline & No & $5(100.0 \%)$ & $117(83.6 \%)$ & $31(88.6 \%)$ & \\
\hline \multirow[t]{2}{*}{ nausea } & Yes & $0(0.0 \%)$ & $16(11.4 \%)$ & $8(22.9 \%)$ & 0.138 \\
\hline & No & $5(100.0 \%)$ & $124(88.6 \%)$ & $27(77.1 \%)$ & \\
\hline \multirow[t]{2}{*}{ pain after feeding } & Yes & $2(40.0 \%)$ & $2(1.4 \%)$ & $2(5.7 \%)$ & $*<0.001$ \\
\hline & No & $3(60.0 \%)$ & $138(98.6 \%)$ & $33(94.3 \%)$ & \\
\hline \multirow[t]{2}{*}{ leakage } & Yes & $5(100.0 \%)$ & 25 (17.9\%) & $11(31.4 \%)$ & $*<0.001$ \\
\hline & No & $0(0.0 \%)$ & $115(82.1 \%)$ & $24(68.6 \%)$ & \\
\hline \multirow[t]{2}{*}{ tube revision } & Yes & $0(0.0 \%)$ & $7(5.0 \%)$ & $3(8.6 \%)$ & 0.612 \\
\hline & No & $5(100.0 \%)$ & $133(95.0 \%)$ & $32(91.4 \%)$ & \\
\hline \multirow[t]{2}{*}{ high residual } & Yes & $2(40.0 \%)$ & $11(7.9 \%)$ & $0(0.0 \%)$ & $* 0.004$ \\
\hline & No & $3(60.0 \%)$ & $129(92.1 \%)$ & $35(100.0 \%)$ & \\
\hline | Length of hospital stay & $3.8 \pm 1.49$ & $7.26 \pm 0.97$ & $27.61 \pm 15.88$ & $* 0.017$ & \\
\hline
\end{tabular}

placement. The choice of procedures depends on patient clinical characteristics and surgeon preference. However, there is no definitive data that proves the benefits of open technique over the laparoscopic [6].

Various complications, ranging from minor to the more severe, have been reported with all methods of placement. Many pediatric patients who undergo gastrostomy tube placement will require long-term enteral therapy. Given the prolonged time pediatric patients may remain enterally dependent, further quality improvement and education initiatives are needed to improve long-term care and outcomes of these patients[7]. Systematic review and meta-analysis of gastrostomy insertion techniques in children revealed percutaneous is associated with an increased risk of major complications when compared to the laparoscopic approach. Advantages in operative time appear outweighed by the increased safety profile of laparoscopic gastrostomy insertion [1].

Gastrostomy tube placement significantly improves the child's physical health, and concomitantly improves the mental health of the child's caregivers, especially at (or after) one year [8]. 
Table 4: Association between outcome of study and clinical characteristics of the patient

\begin{tabular}{|c|c|c|c|c|c|}
\hline & & Type of GT used & & & $P$ - Value \\
\hline Variables & Categories & Foley Cath & Mickey Tube & Mic Tube & \\
\hline \multirow[t]{2}{*}{ Gender } & Male & $68(60.2 \%)$ & $37(66.1 \%)$ & $6(54.5 \%)$ & 0.67 \\
\hline & Female & $45(39.8 \%)$ & $19(33.9 \%)$ & $5(45.5 \%)$ & \\
\hline \multirow[t]{2}{*}{ Concomitant Fundoplication } & Yes & 27 (23.9\%) & $12(21.4 \%)$ & $5(45.5 \%)$ & 0.232 \\
\hline & No & $86(76.1 \%)$ & $44(78.6 \%)$ & $6(54.5 \%)$ & \\
\hline \multirow[t]{4}{*}{ Indication of the procedure } & GERD & $32(28.3 \%)$ & 19 (33.9\%) & $0(0.0 \%)$ & 0.351 \\
\hline & Feeding Intolerance & $4(3.5 \%)$ & $2(3.6 \%)$ & $0(0.0 \%)$ & \\
\hline & Swallowing disorder & $23(20.4 \%)$ & $13(23.2 \%)$ & $4(36.4 \%)$ & \\
\hline & More than one indications & $54(47.8 \%)$ & $22(39.3 \%)$ & $7(63.6 \%)$ & \\
\hline Operative Time & $89.89 \pm 8.77$ & $80.95 \pm 12.54$ & $81.14 \pm 26.82$ & 0.836 & \\
\hline Feeding Start (On day) & $2.11 \pm 0.11$ & $2.02 \pm 1.58$ & $1.80 \pm 0.29$ & 0.649 & \\
\hline \multirow[t]{2}{*}{ Neurological disorder } & Yes & $86(76.1 \%)$ & $43(76.8 \%)$ & 10 (90.9\%) & 0.533 \\
\hline & No & $27(23.9 \%)$ & $13(23.2 \%)$ & $1(9.1 \%)$ & \\
\hline Albumin Level & $25.26 \pm 1.94$ & $28.53 \pm 3.44$ & $16.04 \pm 8.05$ & 0.336 & \\
\hline \multirow{2}{*}{ Complications Post-Operative } & Yes & 35 (31.0\%) & $18(32.1 \%)$ & $6(54.5 \%)$ & 0.28 \\
\hline & No & 78 (69.0\%) & $38(67.9 \%)$ & $5(45.5 \%)$ & \\
\hline \multirow{2}{*}{ lleus } & Yes & $2(1.8 \%)$ & $4(7.1 \%)$ & $1(9.1 \%)$ & 0.154 \\
\hline & No & $111(98.2 \%)$ & 52 (92.9\%) & 10 (90.9\%) & \\
\hline \multirow{2}{*}{ Diarrhea } & Yes & $12(10.6 \%)$ & $15(26.8 \%)$ & $0(0.0 \%)$ & $* 0.008$ \\
\hline & No & $101(89.4 \%)$ & $41(73.2 \%)$ & $11(100.0 \%)$ & \\
\hline \multirow{2}{*}{ Nausea } & Yes & $15(13.3 \%)$ & $9(16.1 \%)$ & $0(0.0 \%)$ & 0.358 \\
\hline & No & $98(86.7 \%)$ & 47 (83.9\%) & $11(100.0 \%)$ & \\
\hline \multirow{2}{*}{ Pain after feeding } & Yes & $6(5.3 \%)$ & $0(0.0 \%)$ & $0(0.0 \%)$ & 0.159 \\
\hline & No & 107 (94.7\%) & $56(100.0 \%)$ & $11(100.0 \%)$ & \\
\hline \multirow{2}{*}{ Leakage } & Yes & $29(25.7 \%)$ & $8(14.3 \%)$ & $4(36.4 \%)$ & 0.136 \\
\hline & No & $84(74.3 \%)$ & $48(85.7 \%)$ & $7(63.6 \%)$ & \\
\hline \multirow{2}{*}{ Tube revision } & Yes & $10(8.8 \%)$ & $0(0.0 \%)$ & $0(0.0 \%)$ & $* 0.043$ \\
\hline & No & $103(91.2 \%)$ & $56(100.0 \%)$ & $11(100.0 \%)$ & \\
\hline \multirow{2}{*}{ High residual } & Yes & $10(8.8 \%)$ & $3(5.4 \%)$ & $0(0.0 \%)$ & 0.451 \\
\hline & No & $103(91.2 \%)$ & $53(94.6 \%)$ & $11(100.0 \%)$ & \\
\hline Length of hospital stay & $6.99 \pm 0.96$ & $9.49 \pm 1.99$ & $46.09 \pm 3.75$ & $* 0.001$ & \\
\hline \multirow{3}{*}{ Outcome } & Death & $3(2.7 \%)$ & $1(1.8 \%)$ & $1(9.1 \%)$ & 0.639 \\
\hline & Full Recovery & $88(77.9 \%)$ & $43(76.8 \%)$ & $9(81.8 \%)$ & \\
\hline & Recovery with sequelae & $22(19.5 \%)$ & $12(21.4 \%)$ & 1 (9.1\%) & \\
\hline
\end{tabular}

Pediatric patients who are the candidates for gastrostomy generally must have normal or near-normal gastric motility, including gastric emptying time as well as small bowel motility [9]. Studies revealed that laparoscopic technique reduces gastro esophageal reflux disease in neurologically impaired patients by improving gastric emptying [10]. Mostly encountered complication were granulation tissue and tube dislodgement after gastrostomy placement in children [11]. However, study conducted in our institution showed high rate of the leakage, pain after feeding and nausea associated with open technique in comparison to the laparoscopic.
Due to the increased negative outcomes including unintentional tube dislodgements, returns to the emergency department, and need for reoperation within 30 days for the patients who underwent open Stamm gastrostomy placement upon retrospective studies it worth to conduct the prospective analysis to confirm that minimally invasive technique is associated with less complicaitons [12].

Laparoscopic and open techniques for Nissan fundoplication with gastrostomy placement have been found safe and appropriate treatment methods with equivalent operating times for the treatment of gastro esophageal reflux in the neonatal intensive 
care unit population [13]. Study showed of the naso-jejunal tubes placement in $94 \%$ of the patients in an average time of 12 minutes [14].

Our research revealed that insertion of gastrostomies by operative techinque takes longer, open or laparoscopic including concomitant fundoplication, approximately 84-90 minutes. No study has been conducted to confirm whether open is associated with an increased risk of major complications when compared to the laparoscopic approach in kids apart from neonatal aged ones.

Postoperative complications like leakage, postoperative pain and nausea prevailed in children more than 1 year old who underwent open technique. The gastrostomy tube replacement using Foley's catheters instead of the commercial gastrostomy ones is a safe and convenient practice without any severe complications [15]. Enteral nutrition in pediatric population increases significantly growth within 6-24 months after insertion of gastrostomy tubes [16].

\section{IRB APPROVAL}

King Fahad Medical City Institutional Review Board

IRB No. 15-425

\section{Consent}

All patients provided informed consent for undergoing the procedures

\section{Conclusion}

Although the technical and clinical outcomes for open and laparoscopic tube placement appear comparable, laparoscopic technique is associated with shorter length of stay and fewer complications.

\section{Competing interests}

The authors declare that they have no competing interests. 


\section{References}

1 Baker L, Beres AL, Baird RA (2015) Systematic review and metaanalysis of gastrostomy insertion techniques in children. J Pediatr Surg. 50:718-725.

2 Villalona G, Mckee M, Diefenbach K (2011) Modified laparoscopic gastrostomy technique reduces gastrostomy tract dehiscence. J Laparo Endosc Adv Surg Tech A 21:355-359

3 Stamm M. Gastrostomy by a new method. Med Newsl 1984; 65.

4 Stey AM, Kenney BD, Cohen ME, Moss RL, Hall BL, et al. (2016) Estimating adverse events after gastrostomy tube placement. Acad Pediatr 16:129-35.

5 Fox D, Campagna EJ, Friedlander J, Partrick DA, Rees DI, et al. (2014) National trends and outcomes of pediatric gastrostomy tube placement. J Pediatr Gastroenterol Nutr 59:582-588.

6 Zamakhshary M, Jamal M, Blair G (2005) Laparoscopic vs percutaneous endoscopic gastrostomy tube insertion: $A$ new pediatric gold standard? J Pediatr Surg; 40:859-862.

7 McSweeney ME, Smithers CJ (2016) Advances in pediatric gastrostomy placement. Gastrointest Endosc. Clin N Am 26: 169-185.

8 Pemberton J, Frankfurter C, Bailey K (2013) Gastrostomy matters-the impact of pediatric surgery on caregiver quality of life. J PediatrSurg 48: 963-970.

9 Itkin M, DeLegge MH, Fang JC (2011) Multidisciplinary practical guidelines for gastrointestinal access for enteral nutrition and decompression from the society of Interventional Radiology and American Gastroenterological Association (AGA) Institute, with endorsement by Canadian Interventional Radiological Association (CIRA) and Cardiovascular and Interventional Radiological Society of Europe(CIRSE). J Vasc Interv Radiol 22: 1089-1106.

10 Kawahara H, Tazuke Y, Soh H, Yoneda A, Fukuzawa M (2014) Does laparoscopy-aided gastrostomy placement improve or worsen gastroesophageal reflux in patients with neurological impairment? J Pediatr Surg. 49: 1742-1745.

11 Naiditch JA, Lautz T, Barsness KA (2010) Postoperative complications in children undergoing gastrostomy tube placement. J Laparoendosc Adv Surg Tech A. 20: 781-785.

12 Sulkowski JP, De Roo AC, Nielsen J, Ambeba E, Cooper JN, et al. (2016) A comparison of pediatric gastrostomy tube placement techniques. Pediatr Surg Int. 32: 269-275

13 Thatch KA, Yoo EY, Arthur LG, Finck C, Katz D, et al. (2010) A comparison of laparoscopic and open Nissen fundoplication and gastrostomy placement in the neonatal intensive care unit population. J Pediatr Surg. 45: 346-349.

14 Patrick PG, Marulendra S, Kirby DF, DeLegge MH (1997) Endoscopic nasogastric-jejunal feeding tube placement in critically ill patients. Gastrointest Endosc 45: 72-76.

15 Kiatipunsodsai S (2015) Gastrostomy tube replacement using Foley's catheters in children. J Med Assoc Thai. 98 Suppl 3: S41-5.

16 Lewis EC, Connolly B, Temple M (2008) Growth outcomes and complications after radiologic gastrostomy in 120 children. Pediatr Radiol 38: 963-970. 